\title{
Matching Recall and Storage in Sequence Learning with Spiking Neural Networks
}

\author{
Johanni Brea, Walter Senn, and Jean-Pascal Pfister \\ Department of Physiology, and Center for Cognition, Learning, and Memory, University of Bern, CH-3012 Bern, Switzerland
}

Storing and recalling spiking sequences is a general problem the brain needs to solve. It is, however, unclear what type of biologically plausible learning rule is suited to learn a wide class of spatiotemporal activity patterns in a robust way. Here we consider a recurrent network of stochastic spiking neurons composed of both visible and hidden neurons. We derive a generic learning rule that is matched to the neural dynamics by minimizing an upper bound on the Kullback-Leibler divergence from the target distribution to the model distribution. The derived learning rule is consistent with spike-timing dependent plasticity in that a presynaptic spike preceding a postsynaptic spike elicits potentiation while otherwise depression emerges. Furthermore, the learning rule for synapses that target visible neurons can be matched to the recently proposed voltage-triplet rule. The learning rule for synapses that target hidden neurons is modulated by a global factor, which shares properties with astrocytes and gives rise to testable predictions.

\section{Introduction}

Increasing experimental evidence in different brain areas shows that precise spike timing can be learned and reliably reproduced over trials. For example, in adult songbirds who learned to repeat the same song, HVC neurons, which are targeting the premotor area RA, reproduce precise spiking patterns during the song production (Hahnloser et al., 2002). In the rat, repeated presentation of a moving spot imprints a stereotypical spiking activity in the visual cortex that can be retrieved after learning (Xu et al., 2012). However, it remains unclear how those spiking patterns can be efficiently learned through synaptic plasticity.

Learning to autonomously reproduce a given spatiotemporal activity pattern is a fundamental challenge approached by the earliest models of recurrent neural networks (Amari, 1972; Hopfield, 1982; Herz et al., 1988; Gerstner et al., 1993; Horn et al., 2000). However, the proposed simple temporal Hebbian rule could be problematic because of its lack of robustness during recall (Hopfield, 1982). Since then, heuristic models for supervised sequence learning in recurrent networks have also been developed for spiking neurons (Ponulak and Kasiński, 2010). All these studies suffer from the same fundamental problem: the synaptic learning rule (storage) is not matched to the neural dynamics (recall) in the sense that the plasticity rule is not derived from first principles that are formulated in terms of neural dynamics.

Another limitation of existing models for sequence learning with spiking neurons (Barber and Agakov, 2002) is the restricted

\footnotetext{
Received Aug. 20, 2012; revised April 3, 2013; accepted April 4, 2013.

Author contributions:J.B., W.S., and J.-P.P. designed research; J.B. and J.P.P. performed research;J.B., W.S., and J.-P.P. wrote the paper.

This research is supported by the Swiss National Science Foundation (J.B. and W.S., Grant 31003A_133094; J.-P.P., Grant PZO0P3_137200) and a grant from the Swiss SystemsX.ch initiative (Neurochoice). We gratefully acknowledge discussions with Robert Urbanczik and Danilo Jimenez-Rezende.

Correspondence should be addressed to Johanni Brea, Department of Physiology, University of Bern, Bühlplatz 5 , CH-3012 Bern, Switzerland. E-mail: johannibrea@gmail.com.

DOI:10.1523/JNEUROSCI.4098-12.2013

Copyright $\odot 2013$ the authors $\quad 0270-6474 / 13 / 339565-11 \$ 15.00 / 0$
}

class of spiking patterns that can be produced with only visible neurons, i.e., neurons that receive the supervising signal. One possible solution is to include a reservoir of hidden neurons, which do not receive a supervised teaching signal, but the synaptic weights between those neurons are usually fixed (Maass and Markram, 2002). Other approaches, such as the Boltzmann machine (Ackley et al., 1985), Helmholtz machine (Dayan et al., 1995), or their extensions to the temporal domain (Hinton et al., 1995; Sutskever et al., 2009) consider learning rules for the weights toward hidden neurons, but the biological plausibility of those rules is open to discussion.

Here we study a general framework with both visible and hidden neurons. In a local neural circuit, neurons that may receive strong input (teaching signal) from outside the circuit are considered to be visible, and neurons that receive only input from neurons inside the circuit are considered to be hidden. We propose a generic synaptic learning rule that is matched to the neural dynamics and that can be adapted to a wide range of neuron models. The learning rule minimizes an upper bound on the Kullback-Leibler (KL) divergence from the target spiking distribution to the distribution produced by the network. This learning rule is consistent with spike-timing dependent plasticity (STDP; Bi and Poo, 1998). The match of recall and storage appears as an explicit link between the time constants in the learning window and the neural time constants. Furthermore, the plasticity rule for synapses that target visible neurons is consistent with the voltage-triplet rule (Clopath et al., 2010). Finally, beside the presynaptic and postsynaptic components, the learning rule for synapses that target hidden neurons is modulated by a global factor. Interestingly, this global factor shares common properties with astrocytes.

\section{Materials and Methods}

Neuron model. We consider a recurrent network of $N$ spiking neurons over a duration of $T$ time bins. Spiking of neuron $i$ is characterized by the spike train $x_{i}$, with $x_{i}(t)=1$ if a spike is emitted at time step $t$, and $x_{i}(t)=0$ 
otherwise. The membrane potential of neuron $i$ is described as in the spike-response model (Gerstner and Kistler, 2002):

$$
u_{i}(t)=u_{0}+\sum_{j=1}^{N} w_{i j} x_{j}^{\varepsilon}(t)+x_{i}^{\kappa}(t)
$$

where $w_{i j}$ is the synaptic strength from neuron $j$ to neuron $i, x_{k}^{\alpha}(t)=$ $\sum_{s=1}^{\infty} \alpha(s) x_{k}(t-s)$ represents the convolution of spike train $x_{k}$ with kernel $\alpha$ and $u_{0}$ is the resting potential. The postsynaptic kernel is characterized by $\varepsilon(s)=\delta_{\mathrm{s}, 1}$ for the one time step response kernel scenarios of Figures 2 and 3, whereas in Figure 4 it is given by $\varepsilon(s)=\left(\exp \left(-s / \tau_{1}\right)-\exp (-s /\right.$ $\left.\left.\tau_{2}\right)\right) /\left(\tau_{1}-\tau_{2}\right)$ for $s \geq 0$ and the adaptation kernel is characterized by $\kappa(s)=c \exp \left(-s / \tau_{r}\right)$ for $s \geq 0$, with both kernels vanishing for $s<0$. For the clarity of the exposition, we chose such a simple neural model description. Note, however, that almost any neural model could be considered (e.g., conductance-based models). The only constraint is that the dynamical model should be linear in the weights, i.e., any dynamical model of the form $\dot{u}_{i}=f_{i}\left(u_{i}\right)+\Sigma_{j} w_{i j} g_{i j}\left(u_{i}, x_{j}\right)$ is suitable.

Consistently with the stochastic spike-response model or equivalently the generalized linear model (GLM; Pillow and Latham, 2008), noise is modeled by stochastic spiking given the (noise-free) membrane potential $u$ in Equation 1, i.e., the probability that neuron $i$ emits a spike in time bin $t$ is a function of its membrane potential: $P\left(x_{i}(t)=1 \mid u_{i}(t)\right)=\rho\left(u_{i}(t)\right)$. We stress the fact that given its own membrane potential, the spiking process is conditionally independent of the spiking of all the other neurons at this time. Due to this conditional independence, the probability that the network with (recurrent) weight matrix $w$ is generating the spike trains $x=\left(x_{1}, \ldots, x_{N}\right)$ can be calculated explicitly as the product of the probabilities for each individual spike train, hence a product of factors $\rho\left(u_{i}(t)\right)$ and $\left(1-\rho\left(u_{i}(t)\right)\right.$, depending on whether neuron $i$ did or did not fire at time $t$, respectively. Abbreviating $\rho_{i}(t)=\rho\left(u_{i}(t)\right)$, this amounts for the log-likelihood (Pfister et al. (2006)) as follows:

$$
\log P_{w}(x)=\sum_{t=1}^{T} \sum_{i=1}^{N} x_{i}(t) \log \rho_{i}(t)+\left(1-x_{i}(t)\right) \log \left(1-\rho_{i}(t)\right)
$$

Unless mentioned otherwise, the firing probability will be assumed to be a sigmoidal $\rho(u)=1 /(1+\exp (-\beta u))$, with parameter $\beta$ controlling the level of stochasticity. We introduced this parameter for convenience: for given weights $w$, the stochasticity of the network can be varied by changing the parameter $\beta$, which multiplies the weights. In the limit $\beta \rightarrow \infty$ each neuron acts like a threshold unit and therefore makes the network deterministic.

Learning task. We separate our $N$ neurons into disjoint classes of $N_{\mathrm{v}}$ visible and $N_{\mathrm{h}}$ hidden neurons. Correspondingly, the spike trains $x$ are separated into those generated by the visible and hidden neurons, $x=(v, h)$. We assume that learning in the recurrent network consists of adapting all the synaptic weights $w_{i j}$ between and among the two types of neurons such that the KL divergence

$$
\mathcal{D}\left(P^{\star}(v) \| P_{w}(v)\right)=\left\langle\log \frac{P^{\star}(v)}{P_{w}(v)}\right\rangle_{P^{*}(v)},
$$

from the target distribution $P^{\star}(v)$ to the model distribution $P_{w}(v)$ of the spike trains of visible neurons becomes as small as possible (Fig. 1). Gradient descent would amount to change the synaptic strength proportionally to the gradient of the negative KL divergence in Equation 3,

$$
\Delta w_{i j} \propto\left\langle\frac{\partial}{\partial w_{i j}} \log P_{w}(v, h)\right\rangle_{P_{w}(h \mid v) P^{*}(v)},
$$

using the fact that $\partial / \partial w_{i j} \log P_{w}(v)=\Sigma_{h} P_{w}(h \mid v) \partial / \partial w_{i j} \log P_{w}(v, h)$. Unfortunately, sampling the sequences of hidden states given a sequence of visible states as suggested by Equation $4, h \sim P_{w}(h \mid v)$, is tricky, since a certain hidden state at time $t$ should be consistent with all visible states, in particular those at later points in time. How to select such hidden states without violating causality is unclear. To circumvent this problem, we suggest minimizing instead an upper bound of the KL divergence. In an alternative approach, using a Monte Carlo Markov Chain to sample from $P_{w}(h \mid v)$, Mishchenko and Paninski (2011) propose sampling by forward-backward algorithm from the conditional distribution of one hidden neuron spike train given all the other spike trains. It is, however, unclear how this can be implemented in biologically plausible neural networks.

Learning by minimizing an upper bound on the KL divergence. To define a biologically plausible sampling procedure we make use of the fact that the firing of each neuron is independent of the activity in the other neurons given the past (cf., Eq. 2). This allows us to factorize the probability for visible and hidden spike sequences into $P_{w}(v, h)=R_{w}(v \mid h) Q_{w}(h \mid v)$, where

$$
\begin{aligned}
& R_{w}(v \mid h)=\prod_{i=1}^{N_{v}} \prod_{t=1}^{T} P\left(v_{i}(t) \mid x(t-1), x(t-2), \ldots\right) \text { and } \\
& Q_{w}(h \mid v)=\prod_{i=1}^{N_{h}} \prod_{t=1}^{T} P\left(h_{i}(t) \mid x(t-1), x(t-2), \ldots\right) .
\end{aligned}
$$

The factor $Q_{w}(h \mid v)$ describes the probability of a hidden activity pattern, when only considering the past hidden and visible activity in each time step. Note that in general $Q_{w}(h \mid v) \neq P_{w}(h \mid v)=P_{w}(v, h) / \Sigma_{h} P_{w}(v, h)$, since in $P_{w}(h \mid v)$ the whole visible activity pattern (past and future) is considered in each time step. Similarly, $R_{w}(v \mid h)$ describes the probability of a visible activity pattern when considering only the past. To obtain samples $h \sim Q_{w}(h \mid v)$, one runs the natural dynamics for the hidden neurons (Eq. 1 including stochastic spiking) while the visible neurons are clamped to $v \sim P^{\star}(v)$. Based on this sampling procedure we introduce the upper bound $\mathcal{F}$ of the KL divergence,

$$
\begin{aligned}
\mathcal{F}\left(P^{\star}(v) \| P_{w}(v)\right) & \\
=\left\langle\log \frac{P^{\star}(v)}{R_{w}(v \mid h)}\right\rangle_{Q_{w}(h \mid v) P^{*}(v)} & \left.\geq \mathcal{D}\left(P^{\star}(v)\right) \| P_{w}(v)\right) .
\end{aligned}
$$

To prove the inequality we note that $P_{w}(h \mid v)=R_{w}(v \mid h) Q_{w}(h \mid v) / P_{w}(v)$. Using the definition and positiveness of the KL divergence we find that $0 \leq \mathcal{D}\left(Q_{w}(h \mid v) \| P_{w}(h \mid v)\right)=\log P_{w}(v)-\left\langle\log R_{w}(v \mid h)\right\rangle_{Q_{w}(h v)}$ and conclude that $-\left\langle\log R_{w}(v \mid h)\right\rangle_{Q_{w}(h v)} \geq-\log P_{w}(v)$. Averaging this last inequality across $P^{\star}(v)$ and subtracting the target entropy $H^{\star}=-\langle\log$ $\left.P^{\star}(v)\right\rangle_{P^{\star}(v)}$, we obtain Equation 6 .

Note that due to the KL properties this bound is tight if and only if $Q_{w}(h \mid v)=P_{w}(h \mid v)$. This is the case if either the activity in the hidden neurons has no effect on the visible activity, i.e., $R_{w}(v \mid h)=P_{w}(v)$ and hence $P_{w}(h \mid v)=P_{w}(v, h) / P_{w}(v)=R_{w}(v \mid h) Q_{w}(h \mid v) / P_{w}(v)=Q_{w}(h \mid v)$, or if the dynamics in the hidden neurons is deterministic, i.e., $P_{w}(h \mid v)=Q_{w}(h \mid v)=\delta_{\mathrm{h}, \mathrm{h}(v)}$ for some function $\mathrm{h}(v)$. Note that with $N_{\mathrm{h}}=(k-1) N_{\mathrm{v}}$, any factorizable Markov chain $\Pi_{i} P_{w}\left(v_{i}(t) \mid v(t-1), \ldots\right.$, $v(t-k))$ of order $k$, which can be parameterized by $P_{w}\left(v_{i}(t) \mid v(t-1), \ldots\right.$, $v(t-k)$ ) (cf., Eqs. 1 and 2) is implementable with deterministic dynamics in the hidden neurons: the first group of $N_{\mathrm{v}}$ hidden neurons are driven by the visible neurons such that their activity is the same as the visible activity one time step in the past, the second group of $N_{\mathrm{v}}$ hidden neurons is driven by the first hidden group such that their activity is the same as the visible activity two time steps in the past and so forth, i.e. $h_{(k-1) \mathrm{Nv}+\mathrm{i}}(t)=v_{i}(t-k)$ (see Fig. $\left.3 B\right)$.

Deriving the batch learning rule. The negative gradient of the upper bound $\mathcal{F}$ in Equation. 6 is evaluated to the following:

$$
\begin{aligned}
-\frac{\partial \mathcal{F}}{\partial w_{i j}}= & \left\langle\frac{\partial}{\partial w_{i j}} \log R_{w}(v \mid h)\right\rangle_{Q_{w}(h \mid v) P^{*}(v)} \\
& +\left\langle\left(\log R_{w}(v \mid h)-\bar{r}\right) \frac{\partial}{\partial w_{i j}} \log Q_{w}(h \mid v)\right\rangle_{Q_{w}(h \mid v) P^{*}(v)},
\end{aligned}
$$


where $\bar{r}$ can be any arbitrary constant since $\left\langle\frac{\partial}{\partial w_{i j}} \log Q_{w}(h \mid v)\right\rangle_{Q_{w}(h \mid v)}=$ $\sum_{h} \frac{Q_{w}(h \mid v)}{Q_{w}(h \mid v)} \frac{\partial}{\partial w_{i j}} Q_{w}(h \mid v)=\frac{\partial}{\partial w_{i j}} \sum_{h} Q_{w}(h \mid v)=\frac{\partial}{\partial w_{i j}} 1=0$. Taking advantage of this arbitrariness, the constant $\bar{r}$ can be chosen to minimize the variance of the second term in Equation 7. Practically, this will be approximated by $\bar{r} \approx\left\langle\log R_{w}(v \mid h)\right\rangle_{Q_{w}(h \mid v) P^{*}(v)}$. To justify this choice we note that the optimal value for $\bar{r}$ can be found as follows: let $e=\frac{\partial}{\partial w_{i j}} \log Q_{w}(h \mid v),\langle e\rangle=$ $0, \operatorname{Var}((r-\bar{r}) e)=\left\langle r^{2} e^{2}-2 \bar{r} \bar{r} e^{2}+\bar{r}^{2} e^{2}\right\rangle-(\langle r e\rangle-\langle\bar{r} e\rangle)^{2} \Rightarrow$ $\frac{\partial}{\partial \bar{r}} \operatorname{Var}((r-\bar{r}) e)=-2\left\langle r e^{2}\right\rangle+2 \bar{r}\left\langle e^{2}\right\rangle=0 \Rightarrow \bar{r}=\frac{\left\langle r e^{2}\right\rangle}{\left\langle e^{2}\right\rangle}$. If $\left\langle r e^{2}\right\rangle \approx\left\langle e^{2}\right\rangle$ one can approximate $\bar{r} \approx\langle r\rangle$. Note that in contrast to Equation 4 , the hidden spike sequences in Equation 7 can now be naturally sampled.

To deduce from Equation 7 a learning rule of the form $\Delta w_{i j} \propto-\partial \mathcal{F} / \partial w_{i j}$ we first have to distinguish between weights $w_{i j}$ projecting onto visible neurons $\left(i=1, \ldots, N_{\mathrm{v}}\right)$, which we call visible weights and weights projecting onto hidden neurons $\left(i=N_{\mathrm{v}}+1, \ldots, N\right)$ - the hidden weights. Due to the conditioning, $R_{w}(v \mid h)$ does not depend on the hidden weights and $Q_{w}(h \mid v)$ does not depend on the visible weights (see Eq. 5). Hence, if the postsynaptic neuron $i$ is visible, the second term in Equation 7 vanishes, and if it is hidden, the first term vanishes. Given the explicit form of the log-likelihoods $\log R_{w}(v \mid h)$ and $\log Q_{w}(h \mid v)$ as in Equation 2 we can directly take the derivatives in Equation 7 and obtain the batch learning rule as follows:

$$
\begin{aligned}
\Delta w_{i j}^{\text {batch }}=\eta \sum_{t=1}^{T} g_{i}(t)\left(x_{i}(t)-\right. & \left.\rho_{i}(t)\right) x_{j}^{\varepsilon}(t) \\
& \cdot\left\{\begin{array}{cc}
1 & \text { if } i \text { visible } \\
\left(\log R_{w}(v \mid h)-\bar{r}\right) & \text { if } i \text { hidden }
\end{array}\right.
\end{aligned}
$$

where $\eta$ is the learning rate and $g_{i}(t)=\frac{\rho_{i}^{\prime}(t)}{\rho_{i}^{\prime}(t)\left(1-\rho_{i}(t)\right)}$, with $\rho_{i}^{\prime}(t)=d \rho(u) /\left.d u\right|_{u=u_{i}(t)}$. For the sigmoidal transfer function $\rho(u)=$ $1 /(1+\exp (-\beta u))$, which is a reasonable and common choice when dealing with binary neurons, the prefactor $g_{i}(t)$ equals $\beta$, since $d \rho(u) /$ $d u=\beta \rho(u)(1-\rho(u))$. In batch learning (Eq. 8) the weights are adapted only after the presentation of several spike patterns $v$. The visible neurons are clamped with spike trains sampled from the distribution $v \sim P^{\star}(v)$, and the hidden neurons follow the neural dynamics, $h \sim Q_{w}(h \mid v)$. As can be seen in Equation 8, the learning rule is different for visible synapses and for hidden synapses. This stands in contrast to our previous work (Brea et al., 2011), where the learning rule is identical for both types of synapses.

In the absence of hidden neurons the learning rule is identical to the one proposed in Pfister et al. (2006) for feedforward networks. The generalization to recurrent networks with hidden neurons, which we consider here, is feasible because of the conditional independence of firing (Eqs. 2 and 5). It should be noted that, even though the learning problem was formulated as "minimize the KL divergence from target to model distribution," this minimization can be implemented with synapses that have only access to local information (presynaptic: $x_{j}^{\varepsilon}(t)$, postsynaptic: $\left.g_{i}(t)\left(x_{i}(t)-\rho_{\mathrm{i}}(t)\right)\right)$ and in the case of hidden synapses one global signal $\left(\log R_{w}(v \mid h)-\bar{r}\right)$. We will assume that each synapse has direct access to the postsynaptic spiking information $x_{i}(t)$ as well as the probability of spiking $\rho_{i}(t)$.

On-line learning rule. To obtain an on-line learning rule, which updates the synaptic weights at each time step, we need to replace the temporal summations in the batch rule (Eq. 8) by leaky integrators. For the synapse-specific part in Equation 8 this leads to the synaptic eligibility trace

$$
e_{i j}(t)=\left(1-\gamma_{1}\right) e_{i j}(t-1)+\gamma_{1} g_{i}(t)\left(x_{i}(t)-\rho_{i}(t)\right) x_{j}^{\varepsilon}(t),
$$

with $\gamma_{1}=1 / T$. Similarly, $\log R_{w}(v \mid h)$ is replaced by

$$
\begin{aligned}
r(t)=\left(1-\gamma_{1}\right) r(t-1)+ & \gamma_{1} \sum_{i=1}^{N_{v}} v_{i}(t) \log \rho_{i}(t) \\
& +\left(1-v_{i}(t)\right) \log \left(1-\rho_{i}(t)\right) .
\end{aligned}
$$

Note that $\log R_{w}(v \mid h)$ is given by the expression in Equation 2 with the summation over neurons from 1 to $N$ replaced by a summation over visible neurons 1 to $N_{\mathrm{v}}$. The identical time constant $\gamma_{1}=1 / T$ of the leaky integrators in Equation 9 and in Equation 10 reflects the fact that in Equation 8 the terms $g_{i}(t)\left(x_{i}(t)-\rho_{\mathrm{i}}(t)\right) x_{j}^{\varepsilon}(t)$ are summed over $t=1, \ldots$, $T$ and so are the terms in $\log R_{w}(v \mid h)$ (cf., Eq. 2). Finally, the term $\left\langle\log R_{w}(v \mid h)\right\rangle_{Q_{w}(h \mid v) P^{*}(v)}$ can be estimated as follows:

$$
\bar{r}(t)=\left(1-\gamma_{2}\right) \bar{r}(t-1)+\gamma_{2} r(t-1),
$$

where the time constant $\gamma_{2}$ of this leaky integrator is much larger than $\gamma_{1}$ to estimate the average $\left\langle\log R_{w}(v \mid h)\right\rangle_{Q_{w}(h \mid v) P^{*}(v)}$. With those dynamic quantities, the synaptic learning rule in Equation 8 now becomes an on-line rule of the form

$$
\delta w_{i j}(t)=\eta e_{i j}(t) \cdot\left\{\begin{array}{cl}
1 & \text { if } i \text { visible } \\
(r(t)-\bar{r}(t)) & \text { if } i \text { hidden }
\end{array} .\right.
$$

In Equation 9 the term $g_{i}(t)\left(x_{i}(t)-\rho_{i}(t)\right)$ corresponds to the postsynaptic and $x_{j}^{\varepsilon}(t)$ to the presynaptic contribution to the weight change. The product of these two terms is low-pass filtered (Eq. 9) to form the eligibility trace, which in the case of hidden neurons is multiplied by the global factor $(r(t)-\bar{r}(t))$ (see Eq. 12). It is interesting to note that the global factor $(r(t)-\bar{r}(t)) \simeq\left(\log R_{w}(v \mid h)-\left\langle\log R_{w}(v \mid h)\right\rangle\right)$ can be seen as an "internal reward signal", which depends on how much more than the average a given hidden activity $h$ helps to produce the visible activity $v$. We call the signal internal, since it is provided by the visible neurons and not by an external teacher.

Initial condition of the dynamics. In the derivation of the learning rule we assumed that $\rho_{i}(t)$ (see Eq. 2 and surrounding paragraph) is given at every moment in time $t$. For times $t$ larger than the neural time constant, $\rho_{i}(t)$ is fully determined by the spiking activity of the network. However, at the beginning of each pattern presentation or recall, $\rho_{\mathrm{i}}(t)$ would also be influenced by spikes that occurred earlier, i.e., before time $t=1$. In practice, for patterns with only visible neurons we took the spikes of the last pattern presentation or recall to initialize the dynamics. In systems with hidden units the whole system was effectively clamped to a predefined spatiotemporal pattern before each pattern presentation or recall, except in Figure 2E, where the system also converged without reset to the initial state.

Recall. Recall means sampling from the model distribution $P_{w}(x)$ and in particular that the visible activity patterns are distributed as $P_{w}(v)$. Therefore, the learning rule minimizes the upper bound $\mathcal{F}\left(P_{w}(v) \| P_{w}(v)\right) \geq \mathcal{D}\left(P_{w}(v) \| P_{w}(v)\right)=0$ during recall. If the bound is tight, i.e., $\mathcal{F}\left(P_{w}(v) \| P_{w}(v)\right)=\mathcal{D}\left(P_{w}(v) \| P_{w}(v)\right)=0$, the gradient $\nabla \mathcal{F}\left(P_{w}(v) \| P_{w}(v)\right)$ equals zero, because $\mathcal{F}\left(P_{w}(v) \| P_{w}(v)\right)$ is in a local minimum. Therefore the weight change is zero on average, i.e., $\langle\Delta w\rangle=0$. However, unless $P_{w}(x)$ is deterministic, the variance $\operatorname{Var}(\Delta w)$ is not zero and therefore diffusion takes place. If $\mathcal{F}\left(P_{w}(v) \| P_{w}(v)\right)>\mathcal{D}\left(P_{w}(v) \| P_{w}(v)\right)$ an additional drift is expected to occur. For the simulations in Figure 3 we compared the time it takes to "forget," i.e., drift away from the final $\mathcal{D}$ value by 0.1 bits, with the time it takes to "relearn" the target. For Markovian targets $(k=1)$ learning was $\sim 100$ times faster than forgetting. For non-Markovian targets $(k>1)$, where performance heavily depends on the hidden weights, learning was $\sim 6$ times faster than forgetting.

Linear separability and Markovianity of sequences. In the one time step response kernel scenario of Figure 2 with deterministic target sequences the notion of linear separability and Markovianity proves helpful for classification. Suppose the target sequence requires a neuron $i$ at time $t$ to be active $x_{i}(t)=1\left(\right.$ silent $\left.x_{i}(t)=0\right)$. This means that during recall, neuron $i$ should get a positive (negative) input at time $t$, i.e., $\Sigma_{j} w_{i j} x_{j}(t-1)>0(<0)$. This puts a constraint on the weights $w_{i j}$. If synaptic strengths $w_{i j}$ exist that respect these constraints for all times $t$ and neurons $i$, the sequence is 
called linearly separable. There exist many methods to test linear separability (Elizondo, 2006).

Is the proposed learning rule capable of finding the weights for a linearly separable sequence? The answer is yes. First, since we know that $w^{*}$ exists, the smallest possible divergence from target to model distribution is zero $\mathcal{D}\left(P^{\star}(x) \| P_{w^{*}}(x)\right)=0$. Second, it can be shown that this divergence is convex in the synaptic weights (Barber, 2011). Therefore a suitable stochastic gradient descent will lead to a perfect solution with $\mathcal{D}\left(P^{\star}(x) \| P_{w}(x)\right)=0$.

We call a deterministic sequence Markovian if any state in the sequence depends only on the previous state, i.e., $x(t)=f(x(t-1))$, for some function $f$ and hence $x(t)=x\left(t^{\prime}\right) \Rightarrow x(t+1)=x\left(t^{\prime}+1\right), \forall t, t^{\prime}$. Note that all linearly separable sequences are Markovian: a nonMarkovian sequence contains transitions with the same initial state but different final states, i.e., for some $t$ and $t^{\prime}$ it contains the subsequences ... $x(t) x(t+1) \ldots$ and $\ldots x\left(t^{\prime}\right) x\left(t^{\prime}+1\right) \ldots$ with $x(t)=x\left(t^{\prime}\right)$ but $x(t+1) \neq$ $x\left(t^{\prime}+1\right)$, for which there do not exist any synaptic weights that satisfy the linear separability constraints, since for a neuron $i$ for which, e.g., $1=$ $x_{i}(t+1) \neq x_{i}\left(t^{\prime}+1\right)=0$ there are no $w_{i j}$ such that $0<\Sigma_{j} w_{i j} x_{j}(t)=$ $\Sigma_{j} w_{i j} x_{i}\left(t^{\prime}\right)<0$; hence there is no non-Markovian sequence that is linearly separable and therefore any linearly separable sequence is Markovian.

Markovian sequences that are not linearly separable require appropriate activity states in a hidden layer (Rigotti et al., 2010) such that the whole sequence of visible and hidden states becomes linearly separable. The minimal number of required hidden neurons depends on the problem. But $N_{\mathrm{h}}=T$ hidden neurons are always sufficient, since it is always possible to find $N_{\mathrm{h}}$ linearly separable states in $N_{\mathrm{h}}$ dimensions. Required are synaptic connections from visible to hidden and hidden to visible. In non-Markovian sequences the visible state at time $t$ does not only depend on the visible activity in the previous time step $t-1$ but depends potentially on earlier activity states, i.e., in time steps $t-2, \ldots$ In this case the activity in the hidden layer can be seen as representing the context in that it carries some information about past visible activity states. Consequently non-Markovian sequences require connections between hidden neurons.

Link to the voltage-triplet rule. In the limit of continuous time, the learning rule for visible synapses can be written as a triplet potentiation term ( 2 post, 1 pre) and a depression term ( 1 post, 1 pre):

$$
\dot{w}_{i j}=\eta g_{i}(t) x_{i}(t) x_{j}^{\varepsilon}(t)-\eta \rho_{i}^{\prime}(t) x_{j}^{\varepsilon}(t),
$$

where $x_{i}(t)$ denotes the Dirac spike train of neuron $i, \rho_{i}^{\prime}(t)=d \rho(u) /\left.d u\right|_{u=u_{i}(t)}$ denotes the derivative of the firing intensity function and the prefactor is defined by $g_{i}(t)=\frac{\rho_{i}^{\prime}(t)}{\rho_{i}(t)}$. Note that in continuous time the prefactor has a slightly different form than in discrete time: to arrive at a continuous time description we explicitly introduce the time bin size $\delta t$, set the probability of spiking in one time bin to $\rho_{i}(t) \delta t$, thereby reinterpreting $\rho_{i}(t)$ as a spike density function, and get in the limit of vanishing time bin size $\lim _{\delta t \rightarrow 0} \frac{\rho_{i}^{\prime}(t) \delta t}{\rho_{i}(t) \delta t\left(1-\rho_{i}(t) \delta t\right)}=\frac{\rho_{i}^{\prime}(t)}{\rho_{i}(t)}$ (Pfister et al., 2006; Brea et al., 2011). Interestingly, formulated in this way, the learning rule closely resembles the voltage-triplet rule proposed by Clopath et al. (2010), which is an extension of the pure spike-based triplet rule (Pfister and Gerstner, 2006). The weight change prescribed by the voltage-triplet rule of Clopath et al. (2010), which we will compare with our rule (Eq. 13), can be also written as a post-post-pre potentiation term and a post-pre depression term:

$$
\dot{w}_{i j}=A_{3}\left[u_{i}^{\alpha}(t)-\theta_{1}\right]_{+}\left[u_{i}(t)-\theta_{2}\right]_{+} x_{j}^{\beta}(t)-A_{2}\left[u_{i}^{\gamma}(t)-\theta_{1}\right]_{+} x_{i}(t),
$$

where the notation $[\cdot]_{+}$denotes rectification, i.e., $[x]_{+}=x$, if $x \geq 0$, otherwise $[x]_{+}=0$. The convolution kernels $\alpha, \beta$, and $\gamma$ are exponential decay kernels with time constants $\tau_{\alpha}$ (resp. $\tau_{\beta}, \tau_{\gamma}$ ), e.g., $\alpha(s)=$ $\tau_{\alpha}^{-1} \exp \left(-s / \tau_{\alpha}\right) \Theta(s)$ where $\Theta(s)$ denotes the Heaviside function, i.e., $\Theta(s)=1$ for $s \geq 0$ and $\Theta(s)=0$ otherwise.

The correspondence between our learning rule for visible synapses of Equation 13 and the voltage-triplet rule (Clopath et al., 2010) in Equation 14 becomes tighter under the following observations and assumptions. First, it should be noted that in the voltage-triplet model, the threshold is high such that $\left[u_{i}(t)-\theta_{2}\right]_{+}$is nonzero only at the timing of the spike. Therefore this term can be replaced by our Dirac spike train $x_{i}(t)$. Second, we can easily assume that the response kernel $\varepsilon(s)$ matches the $\gamma(s)$ convolution kernel. Indeed in Clopath et al. (2010), the time constant $\tau_{\gamma}$ (between 15 and $30 \mathrm{~ms}$ ) was fitted to recordings. Third, in the limit of fast time constants $\tau_{\alpha}, \tau_{\gamma} \rightarrow 0$ (which is reasonable since those time constants in Clopath et al., 2010 are smaller than $10 \mathrm{~ms}$ ), the low-pass filtered versions of the membrane potential can be replaced by their instantaneous value: $u_{i}^{\alpha} \rightarrow u_{i}$, (resp. $\left.u_{i}^{\gamma} \rightarrow u_{i}\right)$. Finally, if we choose a gain function given by $\rho(u)=g_{0}[u-\theta]_{+}^{2}+\nu_{0}$, the factor $\rho^{\prime}(u)=2 g_{0}[u-\theta]_{+}$is a rectified linear function consistent with the voltage triplet model and the factor $\rho^{\prime}(u) / \rho(u)=2 g_{0}(u-\theta) /\left(g_{0}(u-\theta)^{2}+\nu_{0}\right) \Theta(u-\theta)$ is close to a rectified linear function for $u \ll \theta+\sqrt{v_{0} / g_{0}}$ since it is zero for $u<\theta$ and starts linearly (with a slope $2 g_{0} v_{0}^{-1}$ ) for $u>\theta$. Note that any rectified polynomial gain function would work as well (with $\rho^{\prime} / \rho$ differentiable at $u=\theta$ for power of the polynomial $p \geq 2$ ).

Simulation details. In all learning curve plots the measures were normalized by the number of visible neurons and time steps. If for all time steps and neurons the probability to be active is $P\left(x_{i}(t)=1\right)=0.5$, which is the case for $u_{i}(t)=0$ and the sigmoidal spike probability function, the normalized KL divergence from target to model distribution equals one bit minus the normalized entropy of the target distribution. In all simulations the initial weights were chosen to be zero, i.e., $w_{0}=0$.

In Figure $2 A$, a deterministic, linearly separable target was learned with: number of neurons $N=N_{\mathrm{v}}=10$, initial weights $w_{i j}=0$, learning rate $\eta=50$, parameter $\beta=0.2$, resting potential $u_{0}=0$, learning phase of 1000 target sequence presentations. In Figure $2 B$ the fraction of learnable patterns $\mu$ was estimated as the mean of the posterior $P\left(\mu \mid D\left(x^{(1)}\right), \ldots, D\left(x^{(l)}\right) \propto \Pi_{i=1}^{l} P\left(D\left(x^{(i)}\right) \mid \mu\right)\right.$ with flat prior $P(\mu)=$ Uniform $([0,1])$, where $D\left(x^{(i)}\right)$ tells whether the sequence $x^{(i)}$ is learnable or not $(l=100)$. The target distributions are delta distributions $P^{(i)}(x)=$ $\delta_{x, x^{(i)}}$, where each $x^{(i)}$ was sampled from a uniform distribution, i.e., $x^{(i)}(t) \sim P\left(x_{j}^{(i)}(t)=1\right)=0.5$. Linear separability, which is the criterion for learnability, was tested with the linear programming method provided by Mathematica (Elizondo, 2006). For the asymmetric Hebb rule, $w_{i j}=1 / T$ $\Sigma_{t}\left(2 x_{i}(t+1)-1\right)\left(2 x_{j}(t)-1\right)$, we tested, whether these weights lead to perfect recall of the sequence. Note that this measure is more stringent than, for example, the average Hamming distance between target and recalled pattern.

In Figure $2 D$ the same procedure as in Figure $2 B$ was applied to temporally correlated target patterns. We used patterns with $N_{\mathrm{v}}=100 \mathrm{neu}-$ rons and $T=5$ time steps. To generate patterns with correlation length $\alpha$, we choose an initial state $x(0)$ with $P\left(x_{i}(0)=1\right)=0.5$ and subsequent states $x(t)$ with probabilities $P\left(x_{i}(t)=x_{i}(t-1) \mid x_{i}(t-1)\right)=2^{-\frac{1}{\alpha+1}}$. This is equivalent to the more intuitive interpretation of the correlation length as the number of time steps needed until the patterns are uncorrelated, i.e., $P\left(x_{i}(\alpha+1)=x_{i}(0) \mid x_{i}(0)\right)=0.5$. Figure $2 E$ shows the attempt of learning a non-Markovian target with: number of neurons $N=N_{\mathrm{v}}=10$, parameter $\beta=0.2$, resting potential $u_{0}=0$, learning rate $\eta=0.1$; batch algorithm: number of neurons $N_{\mathrm{v}}=10, N_{\mathrm{h}}=10$, parameter $\beta=0.1$, learning rate $\eta=0.1$, estimate of $\bar{r}$ and update of weights every 25 target pattern presentation; on-line algorithm: number of neurons $N_{\mathrm{v}}=10, N_{\mathrm{h}}$ $=10$, learning rate $\eta=0.5$, first low-pass filter time constant $\gamma_{1}=1 / 12$, second low-pass filter time constant $\gamma_{2}=1 / 120$, the low-pass filter variables $e_{i j}, r, \bar{r}$ were initialized with zeros. During the first 100 pattern presentations no update of the hidden weights took place to omit transient effects of the low-pass filter dynamics. In the batch simulation the state of the hidden neurons was reset to a given state $h_{1}$ (indicated by the green line) after each target pattern presentation and before each recall. In the on-line simulation the hidden states were never reset. In all simulations the initial weights were set to zero, i.e., $w_{i j}=0$, the learning phase consisted of $2.5 \cdot 10^{4}$ target pattern presentations.

Figure $3 A$ shows target distribution $P^{\star}(v)=P_{w^{*}}(v)$ with $w_{i j}^{*}$ drawn from a normal distribution with mean zero and SD 5, number of visible neurons $N_{\mathrm{v}}=5$, number of hidden neurons $N_{\mathrm{h}}=0$ for target and model with only visible, $N_{\mathrm{h}}=15$ for model with hidden neurons, parameter $\beta=2 / \sqrt{N}$, learning rate for visible weights $\eta_{\mathrm{v}}=4 \cdot 10^{-4}$, learning rate for hidden weights $\eta_{\mathrm{h}}=10^{-4}$, resting potential $u_{0}=0$, initial model 

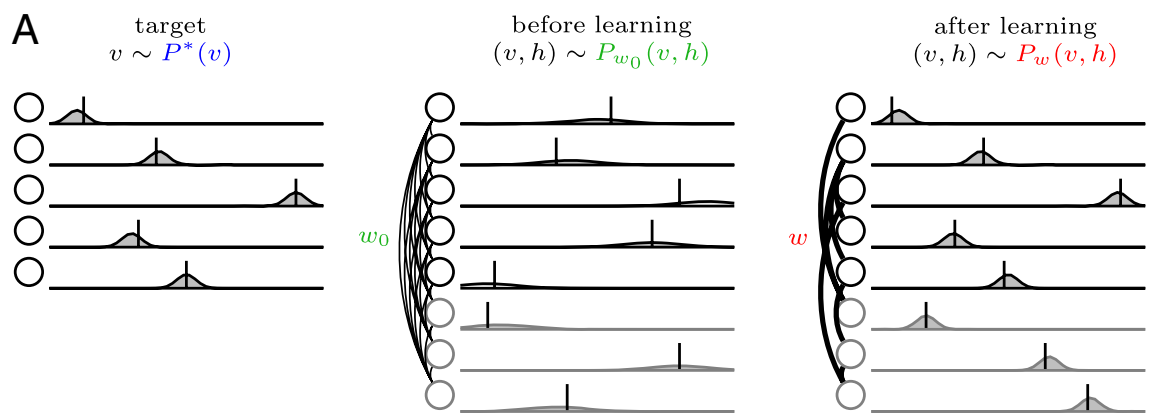

B

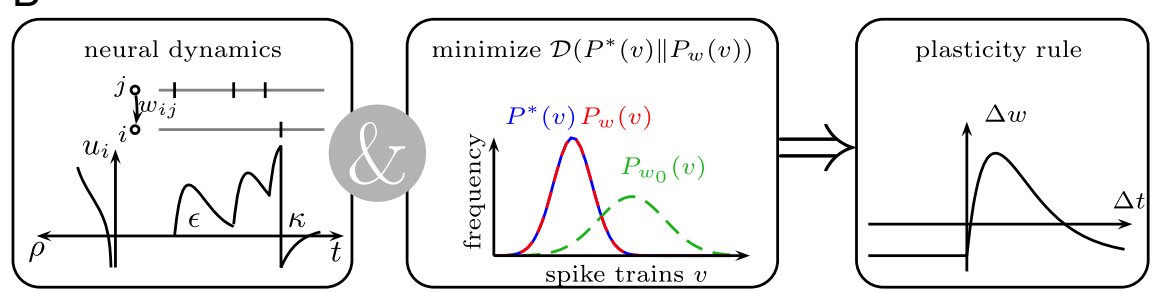

Figure 1. Task and model description. A, Left, Stimuli-evoked activity patterns $v$ (ticks) with probabilities given by a target distribution $P^{*}(v)$ (curves). Middle, In the absence of stimuli the network spontaneously generates activity patterns $x=(v, h)$ distributed according to a model distribution $P_{w_{0}}(x)$ with synaptic strength parameters $w_{0}$. Right, With learning, the distribution of spontaneous activity patterns $P_{w}(v)$ approaches the target distribution. Hidden neurons (in gray) help to support the desired activity patterns in the visible neurons. $\boldsymbol{B}$, Neural dynamics define the model distribution $P_{w}(x)$ trough a spike response kernel $\varepsilon$, an adaptation kernel $\kappa$, and the spike probability $\rho$. Minimizing the divergence $\mathcal{D}\left(P^{*}(v) \| P_{w}(v)\right)$ from the target to the model distribution leads to a plasticity rule for the synaptic strengths $w$ that is matched to the recall dynamics.

weights $w_{0}=0, P\left(x_{0}\right)=\delta_{x_{0}, x^{*}}$. Training data consisted of $10^{7}$ patterns of length 20 time steps, which were generated by the target distribution by running the neural dynamics with target weights $w^{\star}$. The transition frequency data was obtained empirically by generating $10^{4}$ samples of 20 time steps.

Figure $3 B$ shows the same as in Figure $3 A$ but for a different target. The construction of the $k=3$ Markov target is explained in the second paragraph after Equation 6 and in the caption of Figure $3 B$. We excluded target weights $w^{*}$, which parameterize a distribution with highly correlated subsequent visible states, i.e., $\left.\left\langle\left(2 v_{i}(t+1)-1\right)\left(2 v_{i}(t)-1\right)\right\rangle_{t, i, P_{\theta}(x)}\right\rangle$ 0.8 , since such distributions can be accurately approximated with a Markovian model.

Figure $3 C$ shows the KL divergence after learning for different $k$. Results were obtained for 16 different target weight matrices $w^{\star}$ and initial conditions $x^{\star}$. For simulations with static hidden weights the visible to hidden and hidden to hidden weights were the randomly reordered final weights from the corresponding simulation with plastic hidden weights, the initial visible weights were set to zero and trained with the same target.

In Figures 4 and 5 we identify one simulation time step with $1 \mathrm{~ms}$. Figure $4 A$. Number of neurons $N=N_{\mathrm{v}}=100$, response kernel time constants $\tau_{1}=10 \mathrm{~ms}, \tau_{2}=2 \mathrm{~ms}$, adaptation time constant $\tau_{r}=15 \mathrm{~ms}$, adaptation kernel amplitude $c=-10$, parameter $\beta=1 / 3$, learning rate $\eta=0.5$, resting potential $u_{0}=-5$, initial model weights zero, target weights $w_{i j}^{*}=20$, if $i \in\{10(l+1)+1, \ldots 10(l+2)\}$ and $j \in\{10 l+1, \ldots$ $10(l+1)\}, w_{i j}^{*}=-5$, otherwise. Training data consisted of $10^{6}$ states generated by the target distribution by running the neural dynamics with target weights $w^{*}$.

Figure $4 B$ shows the number of neurons $N=N_{\mathrm{v}}=20$, response kernel time constants $\tau_{1}=10 \mathrm{~ms}, \tau_{2}=2 \mathrm{~ms}$, adaptation time constant $\tau_{r}=3 \mathrm{~ms}$, adaptation kernel amplitude $c_{\text {adapt }}=-200$, parameter $\beta=0.1$, learning rate $\eta=100$, resting potential $u_{0}=-5$, initial model weights zero, delta target distribution $P^{\star}(x)=\delta_{x x^{*}}$, with $x^{\star}$ drawn from a distribution with $P\left(\mathrm{x}_{i}^{\star}(t)=\right.$ $1)=0.1$, the learning phase consisted of $2 \cdot 10^{4}$ target pattern presentations. For recall, the system is always initialized in the same way.

Figure $4 C$ shows the number of neurons $N=N_{\mathrm{v}}=10$, fast response kernel kernel time constants $\tau_{1}=15 \mathrm{~ms}, \tau_{2}=7 \mathrm{~ms}$, slow response kernel time constants $\tau_{1}=50 \mathrm{~ms}, \tau_{2}=30 \mathrm{~ms}$, adaptation kernel $\kappa(t)=c_{\mathrm{f}}{ }^{-t / \tau_{f}}+$ $c_{\mathrm{s}}{ }^{-t / \tau_{\mathrm{s}}}$, with $c_{f}=-50, c_{\mathrm{s}}=-5, \tau_{f}=7 \mathrm{~ms}, \tau_{\mathrm{s}}=$ $30 \mathrm{~ms}$, the slow decay with time constant $\tau_{s}$ was added to counteract the influence of the slow response kernel and thus increase the stability during recall, parameter $\beta=0.2$, learning rate $\eta=10, u_{0}=-5$, and initial model weights zero. Target spike trains were generated by injecting strong external positive currents in high firing rate phases and negative currents in low firing rate phases, which we modeled using neural dynamics given by $u_{i}(t)=u_{0}+x_{i}^{k}(t)+$ $u_{\text {ext }}$, where $u_{\text {ext }}=+50(-50)$ in high (low) firing rate phases of the neuron and the sigmoidal spiking firing probability. Each high (low) firing rate phase had a duration of $50 \mathrm{~ms}$. The training phase consisted of $3 \cdot 10^{3}$ samples drawn from the target distribution.

In Figure $5 A$ the change in synaptic strength $\Delta w$ after 50 pairings of one presynaptic with one postsynaptic spike on an interval of $200 \mathrm{~ms}$ was computed for different adaption kernels $\kappa(t)=$ $c e^{-t / \tau_{r}}$ with $\tau_{r}=10 \mathrm{~ms}$ (black: $c=2$, blue: $c=0$, red: $c=-10$ ) while keeping the synaptic response kernel $\varepsilon(t)=\frac{1}{\tau_{1}-\tau_{2}}\left(e^{-t / \tau_{1}}-e^{-t / \tau_{2}}\right)$, with $\tau_{1}=$ $10 \mathrm{~ms}$ and $\tau_{2}=2 \mathrm{~ms}$. The initial value of the membrane potential before stimulation was set to the resting potential $u_{0}=-5$. Initial weight was $w=10$. In Figure $5 B$ the data were fitted with a model given by the probability function $\rho(u)=$ $g_{0}[u-\theta]_{+}{ }^{2}+\nu_{0}$, and response kernel $\alpha(s)$ $=\tau_{\alpha}^{-1} \exp \left(-s / \tau_{\alpha}\right) \Theta(s)$ (see above, Link to the voltage-triplet rule). The fitted parameters are given by the following: $g_{0}=$ $0.94 \cdot 10^{-2} \mathrm{~Hz}, \nu_{0}=7.9 \mathrm{~Hz}, \theta=-36.2, \tau_{\alpha}=41.5 \mathrm{~ms}, w_{0}=1$, and $\eta=0.46$.

All simulations were performed with Mathematica on personal computers, except the simulations in Figure 3, which were written in $\mathrm{C}$ and the fit in Figure $5 B$, which was performed with MATLAB.

\section{Results}

We studied the task of learning to spontaneously produce spiking activity with a given statistics: stimuli make neurons fire in a specific order (Fig. $1 A$, target); in the absence of any stimulus, neurons spike spontaneously; and before learning, the spontaneous activity patterns do not resemble the stimulus-evoked activity patterns (Fig. 1A, before learning). The goal of learning is to change the network dynamics such that after learning the spontaneous activity patterns resemble the stimulus-evoked activity patterns (Fig. $1 A$, after learning). To derive plasticity rules that solve this learning task, we chose the neural dynamics and minimized with respect to synaptic weights a distance measure between stimulus-evoked and spontaneous activity (see Materials and Methods; Fig. $1 B$ ). In this way, the learning rule is matched to the neural dynamics.

To study the proposed learning rules we first elaborate on deterministic target sequences learned with the simplest variant of our model. Deterministic target sequences are of behavioral relevance, since spatiotemporal spike pattern distributions are presumably often sharply peaked in the sense that the typical spike patterns closely resemble each other.

\section{Deterministic target sequences}

In the simplest conceivable form of our model the shape of the postsynaptic kernel $\varepsilon$ is a unit rectangular for one delayed time step and no adaptation takes place. This stochastic McCullochPitts neuron (McCulloch and Pitts, 1943) is of widespread usage 
in artificial neural networks (Baum and Wilczek, 1987; Düring et al., 1998; Barber, 2011).

In Figure $2 A$, target, we show a sequence, which is learnable with only visible neurons. During learning, the stimuli activated the neurons repetitively in the order shown in the figure. After learning, spontaneous activity reproduced the target sequence including the transition from the last to the first spatial activity pattern. This fact is also reflected in the learning curve in Figure $2 A$, which shows that the model distribution approximated the target distribution almost perfectly.

Which sequences are learnable with only visible neurons and which require hidden neurons? Since at each moment in time a neuron can be either active or silent, the total number of visible sequences is $2^{N_{\mathbf{v}} T}$, for a given number of visible neurons $N_{\mathrm{v}}$ and sequence length $T$. We can group these sequences using as criterion either linear separability or Markovianity (see Materials and Methods). It turns out that this grouping helps to formulate minimal architectural requirements, as summarized in Figure 2C: linearly separable sequences can be learned with only visible neurons and synaptic connections between them, Markovian sequences require enough (at most $N_{\mathrm{h}}=T$ ) hidden neurons and at least synaptic connections from visible to hidden and hidden to visible, and non-Markovian sequences demand additionally connections between hidden neurons.

The sequence shown in Figure $2 A$ is linearly separable and thus can be learned with only visible neurons. The sequence in Figure $2 E$ is non-Markovian: the state marked by a blue triangle occurs twice in the sequence. Furthermore, already the second state (red triangle), where all neurons are silent, renders the sequences not linearly separable. This is a consequence of our choice of coding (1, active; 0 , silent) and adaptable parameters (only synaptic weights $w_{i j}$ ), which we used to account for biological constraints. This sequence can only be learned with appropriate recurrent connections of the hidden neurons. We show that both the batch and the on-line learning rule find appropriate synaptic weights by stochastic gradient descent on the upper bound $\mathcal{F}$ of the divergence $\mathcal{D}$ from target to model distribution. This bound becomes tight, i.e., $\mathcal{F}=\mathcal{D}$, toward the end of learning (see Materials and Methods), which is reflected in the diminishing distance between the $\mathcal{F}$ and $\mathcal{D}$ curves in Figure $2 E$.

What is the typical size of the subset of linearly separable, and thus learnable, sequences for given $N_{\mathrm{v}}$ and T? In Figure $2 B$ we

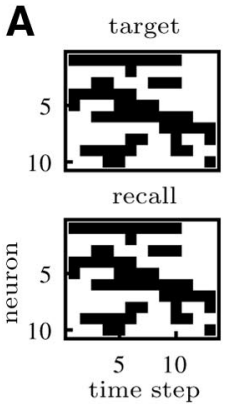

C

E during recall.
$1 \quad 2^{N_{\mathrm{v}} T}$
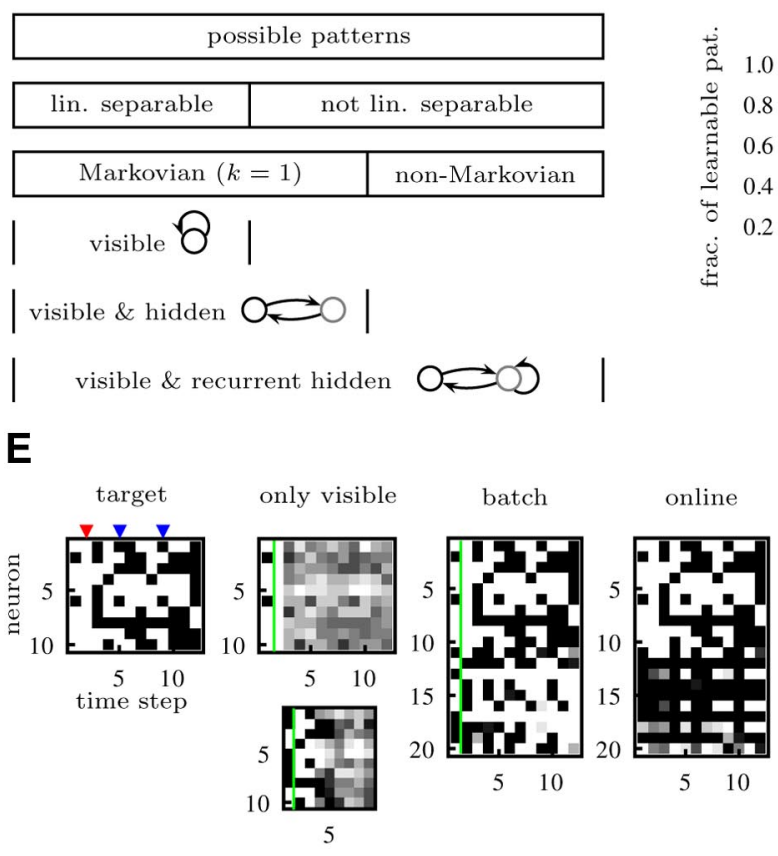

Figure 2. Learnability of deterministic target sequences with a network of McCulloch-Pitts neurons. $\boldsymbol{A}$, Linearly separable sequence can be learned with only visible neurons. $\boldsymbol{B}$, The fraction of learnable sequences with only visible neurons for different relative sequence lengths and number of neurons: in red for a learning rule that finds an optimal solution if the sequence is linearly separable, in blue for the asymmetric Hebb rule (see Results) $\left(N_{v}=40,60,80\right.$, light to dark). The dots indicate the mean, the error bars indicate the SD of the posterior distribution with flat prior. C, For a given number of visible neurons $N_{\mathrm{v}}$ and sequence length $T$, the number of possible deterministic target sequences is $2^{N_{v} T}$. These sequences can be classified by either linear separability or Markovianity (see Materials and Methods). Linearly separable sequences can be learned with only visible neurons; nonlinearly separable but Markovian sequences require sufficient hidden neurons and appropriate synaptic weights from visible to hidden and from hidden to visible neurons, and non-Markovian sequences require additionally connections between hidden neurons. $\boldsymbol{D}$, The simple asymmetric Hebb rule (blue curve; see Results) does often not find weights that allow perfect recall if the pattern is temporally correlated. The fraction of learnable patterns (red curve) decreases only slowly with increasing correlation length $\alpha$. $\boldsymbol{E}$, This target sequence is obviously not linearly separable and even non-Markovian: it contains an activity gap in the second time step (highlighted with red triangle) and in time steps 5 and 9 (highlighted with blue triangles) the same spatial pattern is followed by two different patterns in time steps 6 and 10 . After training, a network with only visible neurons only recalls the gap faithfully when initialized with the first time step and only reproduces the states up to the ambiguous transition faithfully when initialized with the third time step just after the gap. Shown in each case is an overlay of 10 recalls. With the batch algorithm in Equation 8 and the on-line plasticity rule in Equation 12 the sequence can be learned. The states left of the green line were clamped for recall. With the on-line rule, the system learned to periodically produce the sequence and no further clamping of initial states was required

show the fraction of learnable sequences as a function of the relative sequence length $T / N_{\mathrm{v}}$ for different $N_{\mathrm{v}}$. Below a relative sequence length of approximately $T / N_{\mathrm{v}} \approx 1.5$ the probability for the sequence to be linearly separable is very close to 1 . The critical value $T / N_{\mathrm{v}} \approx 1.5$ is again a consequence of our choice of coding. For (1, active; -1 , silent) coding we expect the critical relative 
A
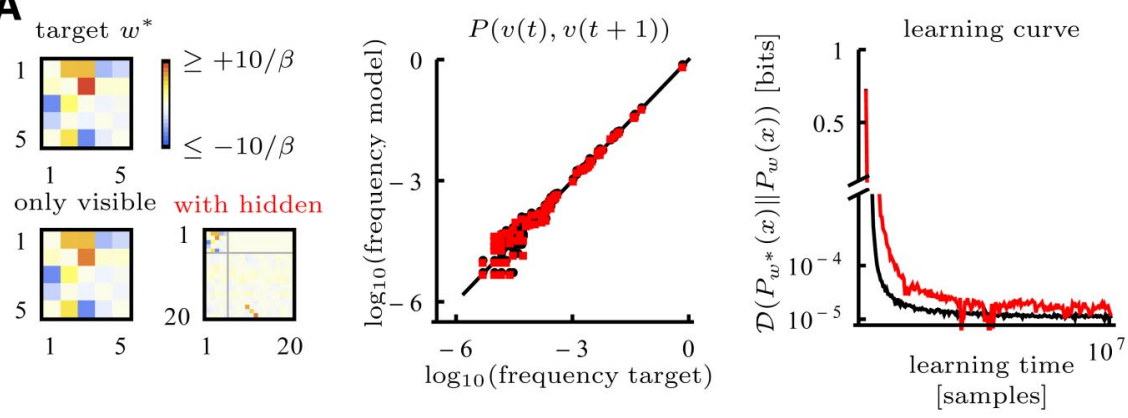

B
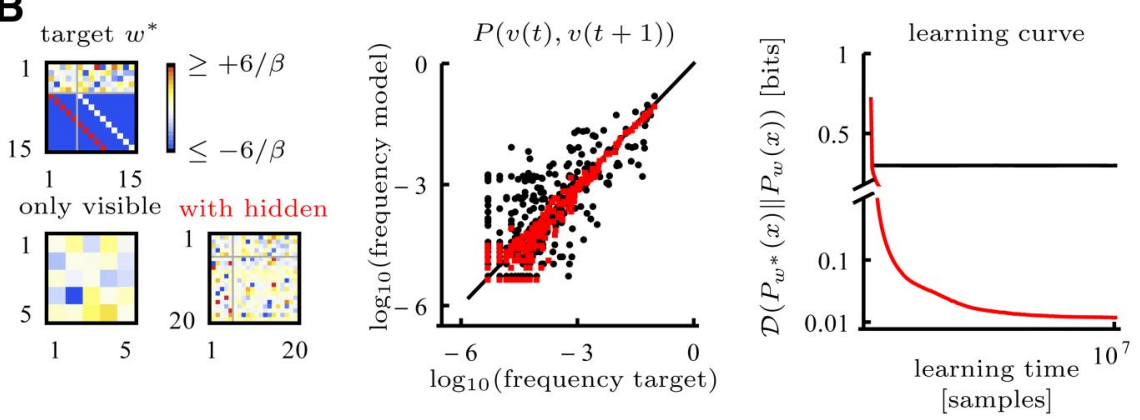

C

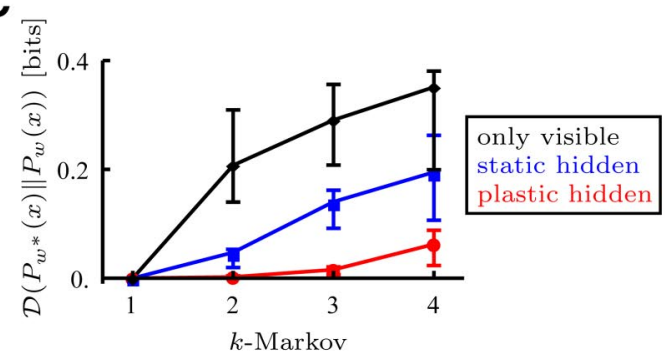

Figure 3. Stochastic targets learned with McCulloch-Pitts dynamics. In all the simulations the target distribution was parameterized by target weights $w^{*} . A$, Left, For a Markovian target with only visible neurons $(k=1)$ the weights can be learned with a model with only visible neurons or a model with visible (1-5) and hidden (6-20) neurons (upper left submatrix: visible to visible connections). Middle, Empirical frequency of subsequent activity patterns $v(t)$ and $v(t+1)$. For dots on the black line the model frequency matches the target frequency. (black, only visible; red, with hidden) Right, The learning curve is similar for both models. $B, A k=3$ target cannot be accurately learned with only visible neurons but can be learned with a model that includes hidden neurons. The target was implemented with connectivities such that the first group of hidden neurons $(6-10)$ receives strong excitatory input from the visible and the second group of hidden neurons (11-15) receives strong excitatory input from the first group of hidden neurons. The learned hidden weights do not need to be the same as the target weights. $\boldsymbol{C}$, With increasing complexity $(k=1$ to $k=4)$ a model with only visible neurons (black) or static hidden weights (blue) is not sufficient to accurately learn the target. The static hidden weights were obtained by reshuffling the weights learned in a simulation with plastic hidden weights. A model with 15 plastic hidden neurons (red) performs well up to the capacity limit $k=4$. Learning stopped after $10^{7}$ target samples, even when learning did not converge. Dots indicate the median, error bars indicate the first and third quartile of the results from 16 simulations with different targets.

sequence length to be at $T / N_{\mathrm{v}} \approx 2$ (Hertz et al., 1991). As a reference we compare this to a simple temporal Hebb rule $\Delta w_{i j}=\frac{1}{T} \Sigma_{t=1}^{T}\left(2 x_{i}(t+1)-1\right)\left(2 x_{j}(t)-1\right)$ as proposed in Hopfield (1982); Düring et al. (1998); Grossberg (1969); Herz et al. (1988); Gerstner et al. (1993); Horn et al. (2000). It is not surprising that this simple temporal Hebb rule, for which linear separability is not a sufficient condition for a pattern to be learnable, in general does not find synaptic weights to perform perfect recall: instead of solving an optimization problem (minimizing the divergence from target to model distribution), which leads to a learning rule that is matched to the dynamics, it is simply inspired by the symmetric Hebb rule used in Hopfield networks (Hopfield, 1982). Its weakness becomes even clearer when we consider correlated patterns (Fig. 2D): the simple temporal Hebb rule performs well only for uncorrelated patterns whereas the probability of linearly separable sequences decreases slowly with increasing correlation.

\section{Stochastic target}

Some stimuli might evoke spike patterns that do not follow a sharply peaked distribution. Instead, the spike patterns look quite different each time a sample from the target distribution $P^{\star}(x)$ is drawn. Even though they do not look similar, there might be some temporal dependencies in the sense that the spiking activity $x(t)$ at time $t$ depends on earlier spiking activity states $x(t-k), k>0$. Variability in the target patterns can arise due to many different reasons. First, there is an extrinsic source of variability, as the same stimulus can be presented with small variations. Second, neurons and synapses are intrinsically noisy, so the neural network that provides the teaching signal will be subject to noise. Interestingly, in the proposed framework the level of noise during recall can be matched to the target variability through synaptic plasticity.

As we showed in Materials and Methods (second paragraph after Eq. 6) it is possible to implement a large class of stochastic target distributions, namely factorizable Markov chains product $P_{w}\left(v_{i}(t) \mid v(t-1)\right.$, $\ldots, v(t-k))$ of order $k$, which can be parameterized by $P_{w}\left(v_{i}(t) \mid v(t-1), \ldots, v(t-k)\right)$ (cf., Eqs. 1 and 2). For Markovian targets $(k=1)$ and a model without hidden neurons, the solution is unique due to the convexity of the KL divergence. In Figure $3 A$ we demonstrate in an example that the learning rule effectively leads to the solution. For $k>1$ a model with only visible neurons fails to learn the target, whereas a model with sufficient hidden neurons equipped with our learning rule succeeds (Fig. 3B). Under the light of the remarkable capabilities of reservoir computing (Maass and Sontag, 2000; Lukoševičius and Jaeger, 2009; Rigotti et al., 2010) it is interesting to note that a random choice of static hidden weights obtained by reshuffling learned weights is not sufficient to learn the target (Fig. 3C).

\section{$\boldsymbol{\alpha}$-shaped kernels}

So far we discussed the plasticity rule for a model with very simple dynamics. However, the neuron model in Equation 1 allows for more realistic dynamics. In the examples shown in Figure 4, a presynaptic spike evokes an $\alpha$-shaped synaptic response $\varepsilon$ (Fig. $4 B)$, which is felt by the postsynaptic neuron, and an adaptation $\kappa$, which prevents immediate follow-up spikes in the presynaptic cell. With this dynamics it is possible to learn a "synfire chain" (Fig. $4 A$ ) or a pattern of precise spike times (Fig. $4 B$ ). Depending on the target distribution and the neural dynamics, timing errors 
may propagate and the pattern eventually becomes unstable (Diesmann et al., 1999). This fading of precision is less problematic with a rate code.

In Figure $4 C$, target, the pattern of Figure $2 A$ is encoded with periods of high and low firing rate. The system can reasonably well learn this target with an $\alpha$-shaped synaptic response $\varepsilon_{1}$ and adaptation kernel $\kappa$ (Fig. $4 C$, model with only $\varepsilon_{1}$, blue learning curve). Fast synaptic responses $\varepsilon_{2}$ help to further stabilize the pattern and increase performance (Fig. 4C, model with both $\varepsilon_{1}$ and $\varepsilon_{2}$, red learning curve). The motivation for using synaptic responses on two different timescales arises from the idea that fast connections establish attractors that encode for the different elements in the sequence and slow responses push the dynamics from one attractor to the next (Sompolinsky and Kanter, 1986). Neurons could implement fast and slow responses to one presynaptic spike with different types of synapses, e.g., fast AMPA synapses and slow NMDA synapses.

\section{Biological plausibility}

When learning is formulated with a computational goal in mind, like minimizing the difference between target and model distribution, it is far from obvious that the resulting learning rule is biologically plausible in the sense that it respects constraints of biological systems and is consistent with experimental data. Here we argue that the proposed learning rule in Equation 12 is biologically plausible.

The learning rule in Equation 12 respects causality, is implemented in an online fashion, and depends on presynaptic and postsynaptic $x$ activity and a modulatory factor for hidden synapses. The prepost term shares similarities with STDP: Figure $5 A$ shows the STDP window predicted by the learning rule ( $\mathrm{Bi}$ and $\mathrm{Poo}$, 1998; Pfister et al., 2006; Brea et al., 2011). Note that this learning rule, which minimizes the divergence in Equation 3 from target to model, predicts that the causal part of the STDP window should be matched to the dynamics of the synaptic transmission. The acausal part depends on the adaptation properties of the neuron. Interestingly, the learning rule is closely related to the voltage-triplet rule (Pfister and Gerstner, 2006; Clopath et al., 2010; see Materials and Methods) and is compatible with the frequency dependence of STDP as observed by Sjöström et al. (2001) (Fig. 5B).

For hidden synapses, the plasticity rule does not depend only on the presynaptic and postsynaptic activity, but is also modulated by a global factor. This global factor could be implemented by astrocytes for mainly three reasons. First, it has been shown that astrocytes, which contact a large number of synapses, can modulate synaptic plasticity (Henneberger et al., 2010; Min and Nevian, 2012). Similarly, in our framework, the semi-global the green line.
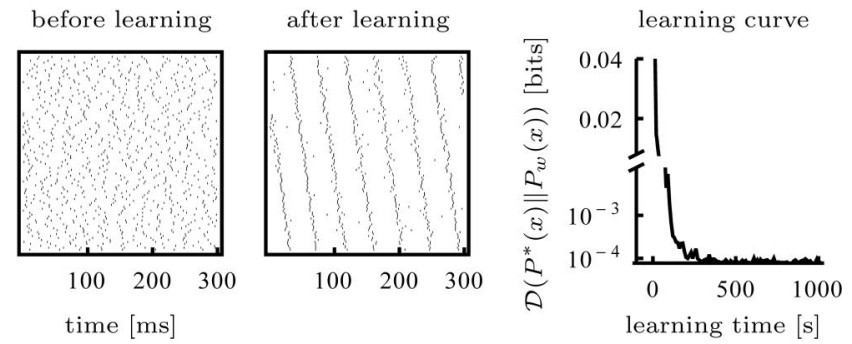
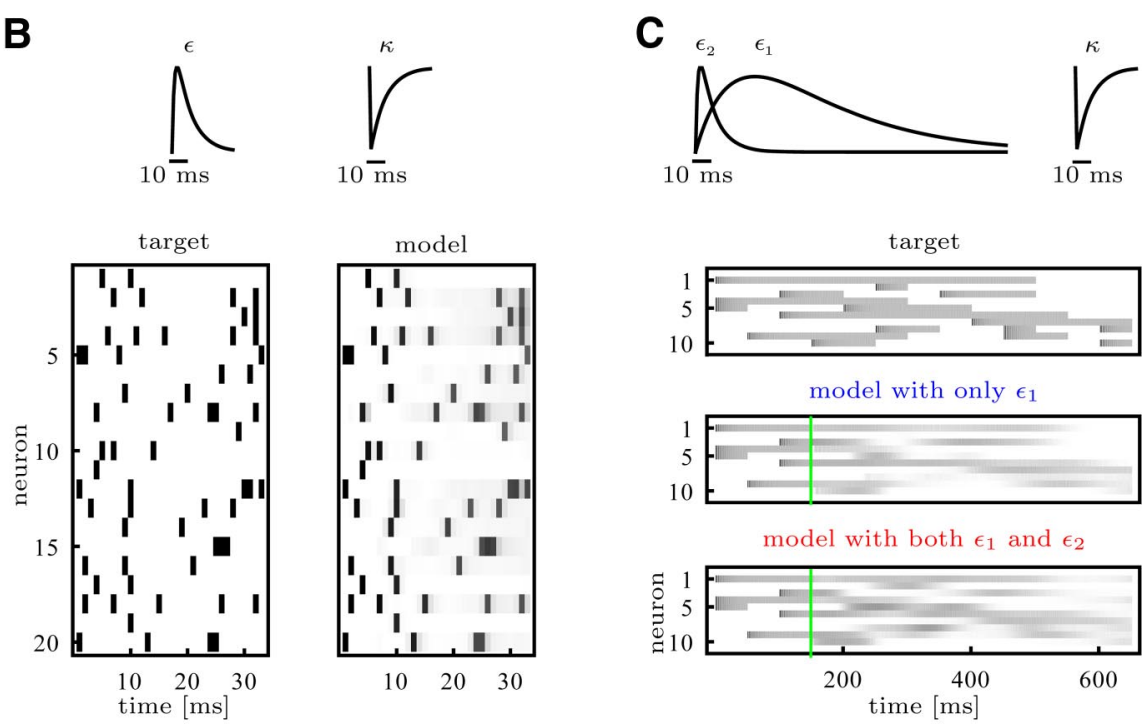

model with only $\epsilon_{1}$

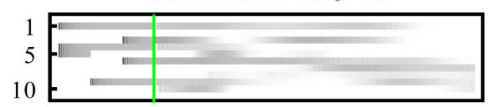

model with both $\epsilon_{1}$ and $\epsilon_{2}$

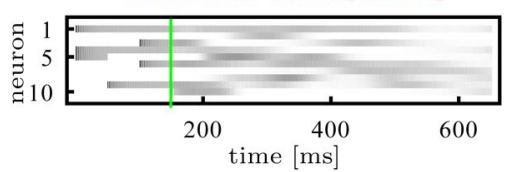

learning curve

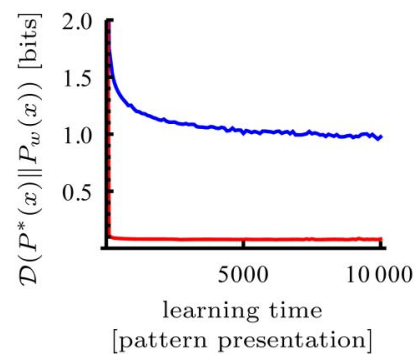

Figure 4. Target distributions learned with $\alpha$-shaped response kernels. $\boldsymbol{A}$, The target distribution $P^{*}(x)=P_{w^{*}}(x)$ has the same parametrization as the model with excitatory weights from groups of 10 neurons projecting to subsequent groups of is encoded with periods of high or low firing rate. The target was generated by applying strong input current square pulses. Refractoriness sets an upper bound to the maximal firing rate. In recalls the model system was clamped to the target up to

modulating factor (see Eq. 12) affects the plasticity of a large group of synapses (the hidden synapses). Second, according to our model, this global factor (see Eq. 12) acts at a slower time constant than the membrane time constant, which is consistent with the calcium dynamics of astrocytes (Di Castro et al., 2011). Finally, a causal pairing (pre-then-post) can lead either to longterm potentiation or long-term depression depending on the sign of the global factor $(r(t)-\bar{r}(t))$. This is in agreement with the study of Panatier et al. (2006) who showed that when D-serine, which is an NMDA co-agonist, is released by astrocytes, longterm depression can be turned into long-term potentiation. Unlike what is suggested by Panatier et al. (2006), this type of sliding threshold $(\bar{r}(t)$ in our case) is conceptually different from the one in the BCM learning rule (Bienenstock et al., 1982) since it is a global quantity whereas in the BCM learning rule the sliding 

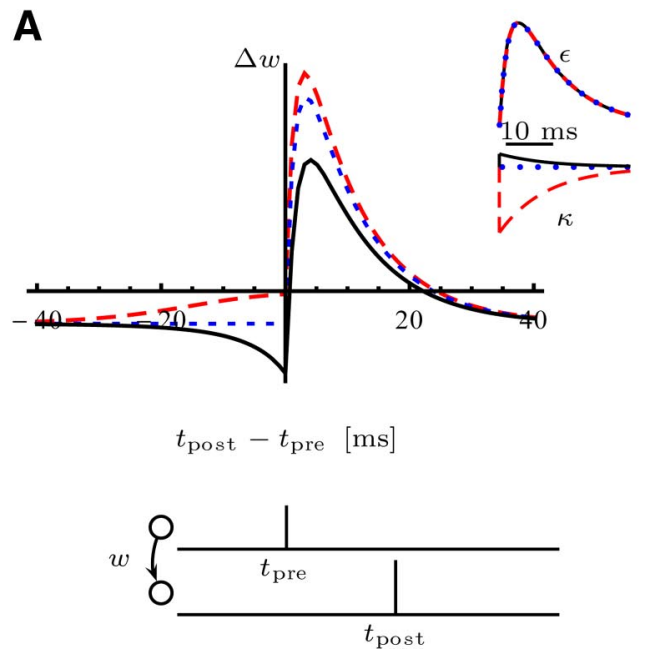

Figure 5. The learning rule shares similarities with STDP. $\boldsymbol{A}$, The STDP window for different adaptation kernels (on the right in black, blue, and red): the synaptic weight change $\Delta w$ of a positive synaptic strength $w>0$ after a pairing protocol on an interval of $200 \mathrm{~ms}$, in which both neurons spike once with a separating interval of $\Delta t=t_{\text {post }}-t_{\text {pre. }}$. Note that the shape of the curve in the causal part $\Delta t \geq 0$ is determined by the response kernel-shape $\varepsilon$ and in the acausal part $\Delta t<0$ depends on the adaptation kernel $\kappa . \boldsymbol{B}$, Experimental (black) and model (red) weight change induced by the pairing protocol described by Sjöström et al. (2001). Every $10 \mathrm{~s}$, bursts of five pairs of presynaptic and postsynaptic spike with a relative timing of $\Delta t=t_{\text {post }}-t_{\text {pre }}=10 \mathrm{~ms}$ (solid lines) and $\Delta t=-10 \mathrm{~ms}$ (dashed line) are induced at different pairing repetition frequencies. Fifteen such bursts are elicited (which give a total of 75 pairs) for all repetition except at $0.1 \mathrm{~Hz}$. At this frequency, only 50 pairs are elicited (Sjöström et al. (2001), their Experimental Procedures). It is assumed that at the beginning of the simulation, the weight is set to 1 and updated only after the induction protocol to mimic the time lag between induction and expression in the experiments.

threshold is a local quantity that depends on the history of the postsynaptic activity. Note that the proposed global factor depends on activity in visible neurons and affects hidden synapses only. Thus, if astrocytes are responsible for this signaling, they "need to know" which neurons are visible and which are hidden. This might be possible due to geometrical arrangement or chemical signaling.

\section{Discussion}

Learning a statistical model for temporal sequences with hidden units is a challenging machine learning task in itself. Here we considered an even harder problem in that we are interested in a biologically plausible learning rule that can solve this task. To be biologically plausible, the learning rule has to be local, causal, and be consistent with experimental data. We derived a biologically plausible learning rule that minimizes by stochastic gradient descent an upper bound of the KL divergence from target to model distribution.

Because the proposed learning rule is minimizing an upper bound of the KL divergence and not the KL divergence itself and because the tightness of the bound is not explicitly controlled, unlike in the Helmholtz framework (Dayan et al., 1995), the maxima of the two different objective functions could in principle be located at different places in the parameter space. It is, however, interesting to note that after some learning time, the bound becomes tighter, as can be seen on Figure $2 E$ where the KL divergence between the proposal distribution $Q_{w}(h \mid v)$ and the posterior distribution $P_{w}(h \mid v)$ decreases. In fact, we can show that at the beginning of learning and at the end of learning of deterministic patterns, the bound is tight. Indeed, at the beginning of the learning all the weights (and in particular the weights toward visible neurons) are initialized to zero and therefore, the function $R_{w}(v \mid h)$ is independent of the hidden activity and thus $\mathcal{D}$ and $\mathcal{F}$ are identical. At the end of learning, for a deterministic pattern, the proposal distribution is a Dirac delta distribution and therefore $\mathcal{D}=\mathcal{F}$.
It is interesting to note that the learning rule for the hidden neurons in Equation 12 appears formally similar to a reward-based learning rule (Pfister et al., 2006), but now the reward is an internally computed quantity and does not depend on an external reward. Loosely speaking, if the hidden units contribute to make the visible spikes likely, the synapses targeting those hidden units receive an internal reward signal $(r(t)-\bar{r}(t))$ (Eq. 12).

The formulation of learning as minimizing the KL divergence (or, equivalently, maximizing the log-likelihood) or an upper bound thereof is common practice in machine learning. The novelty of our approach lies in the specific choice of the upper bound of the KL divergence, which relies on the assumption of conditional independence for the neural firing, i.e., given its membrane potential, the probability of firing of a neuron is conditionally independent of the firing of all other neurons at the same time. Even though this assumption is perfectly reasonable and widely used (e.g., in the GLM framework; Pillow and Latham, 2008), it is the key assumption that allows the joint distribution over visible and hidden activity to be expressed as a product of a distribution from which it is easy to sample $\left(Q_{w}(h \mid v)\right)$ and a function which is easy to calculate $\left(R_{w}(v \mid h)\right)$. This stands in contrast to temporal Boltzmann machines (Sutskever et al., 2009) where this assumption is not made and sampling usually involves running a Monte Carlo Markov Chain in each time step, which is hard to justify under the light of biological plausibility.

Another approach to learn a statistical model of spatiotemporal spike pattern with visible and hidden neurons is a generalization of the expectation-maximization algorithm proposed by Pillow and Latham (2008). Yet, as a version of the Helmholtz machine, the distribution, from which the hidden states are sampled, uses a different parameterization for storage (recognition; wake phase of learning) and recall (generation; sleep phase of learning) and assumes an acausal kernel, which renders the model unsuitable for a biologically realistic implementation. To circumvent the need of sampling the hidden states, $h \sim P_{w}(h \mid v)$ (see Eq. 4), Rezende et al. (2011) proposed to calculate explicitly the expectation under the posterior distribution $P_{w}(h \mid v)$. To achieve this, they had to assume a weak coupling between neurons and then approximate the true posterior distribution by a Gaussian process on the membrane potential. This weak coupling assumption is however difficult to justify at the end of learning where individual weights can become large.

A drawback of the proposed learning rule for systems including hidden neurons is the potentially long learning time. The plasticity rule relies on stochastic gradient ascent by sampling hidden sequences $h \sim Q_{w}(h \mid v)$. As in any gradient descent method, the learning time depends on the learning rate and the initial condition: the learning time is short if at the beginning of learning the weights projecting onto hidden neurons are such that the samples $h \sim Q_{w}(h \mid v)$ help to quickly reduce the difference measure between target and model distribution and can be long otherwise. In some cases it is even 
possible to find initial weights that supersede any further learning of hidden weights. For example, any possible sequence as discussed in Figure $2 C$ could be learned with a (hardwired) delay line of length $T$ with $N_{\mathrm{h}}=T$ hidden neurons and no learning of the hidden weights. Alternatively, the delay line could be implemented by a large enough number of randomly connected hidden neurons (Maass and Sontag, 2000; Lukoševičius and Jaeger, 2009; Rigotti et al., 2010) where the weights are chosen from a given distribution. The number of randomly connected hidden neurons needed might be very large to guarantee good solutions in any case. Since the goal of this paper was rather to demonstrate that the learning rule is capable of learning distributions that could not be learned with only visible neurons, we did not make use of elaborated choices of the initial conditions and started all simulations with initial weights $w_{0}=0$.

Our paper is not the first one to propose the idea that storage and recall should be matched. Indeed Sommer and Dayan (1998) and Lengyel et al. (2005) already proposed that there should be a tight link between the plasticity rule and the neural dynamics. Interestingly their approach is complementary to the one presented here. Indeed, they start from a given plasticity rule and then derive the optimal (Bayesian) recall dynamics for this given plasticity rule. Here we are following the opposite path. We start from the neural dynamics and then derive the plasticity rule. Given the richness and the accuracy of existing neural models and given the absence of a canonical model of synaptic plasticity, we preferred to start from what is well known and derive predictions on what is largely unknown.

An interesting outcome of our model is that the learning rule for hidden synapses does not depend only on the presynaptic and postsynaptic activity, but is also modulated by a global factor. We argued in this paper that this global factor could be provided by astrocytes. To experimentally test this hypothesis we note that the global factor is predicted to depend on the voltage of the visible neurons. In particular, independently of the precise implementation of the model (be it by minimizing an upper bound on the KL divergence as presented here or by directly minimizing KL divergence itself as in Brea et al., 2011), the global factor crucially depends on the presynaptic membrane potential at the time of the spike (see Eq. 10). So the key experimental step would be to show that astrocytic activity depends on the membrane potential of the presynaptic neuron at the time of the spike. This prediction seems plausible since it was found that a depolarization of the presynaptic membrane potential at the time of the spike causes a larger postsynaptic potential (Alle and Geiger, 2006; Shu et al., 2006). We suggest that astrocytes could have a similar sensitivity to the membrane potential at the time of the spike.

\section{References}

Ackley DH, Hinton GE, Sejnowski TJ (1985) A learning algorithm for Boltzmann machines. Cogn Sci 9:147-169. CrossRef

Alle H, Geiger JR (2006) Combined analog and action potential coding in hippocampal mossy fibers. Science 311:1290-1293. CrossRef Medline

Amari SI (1972) Learning patterns and pattern sequences by self-organizing nets of threshold elements. IEEE Trans Comput 21:1197-1206. CrossRef

Barber D (2011) Bayesian reasoning and machine learning. New York: Cambridge UP.

Barber D, Agakov F (2002) Correlated sequence learning in a network of spiking neurons using maximum likelihood. Tech Report EDI-INF-RR0149, School of Informatics, Edinburgh, UK.
Baum EB, Wilczek F (1987) Supervised learning of probability distributions by neural networks. Adv Neural Inf Process Syst 52-61.

Bi GQ, Poo MM (1998) Synaptic modifications in cultured hippocampal neurons: dependence on spike timing, synaptic strength, and postsynaptic cell type. J Neurosci 18:10464-10472. Medline

Bienenstock EL, Cooper LN, Munro PW (1982) Theory for the development of neuron selectivity: orientation specificity and binocular interaction in visual cortex. J Neurosci 2:32-48. Medline

Brea J, Senn W, Pfister JP (2011) Sequence learning with hidden units in spiking neural networks. Adv Neural Inf Process Syst 24:14221430.

Clopath C, Büsing L, Vasilaki E, Gerstner W (2010) Connectivity reflects coding: a model of voltage-based STDP with homeostasis. Nat Neurosci 13:344-352. CrossRef Medline

Dayan P, Hinton GE, Neal RM, Zemel RS (1995) The Helmholtz machine. Neural Comput 7:889-904. CrossRef Medline

Di Castro MA, Chuquet J, Liaudet N, Bhaukaurally K, Santello M, Bouvier D, Tiret P, Volterra A (2011) Local Ca2+ detection and modulation of synaptic release by astrocytes. Nat Neurosci 14:1276-1284. CrossRef Medline

Diesmann M, Gewaltig MO, Aertsen A (1999) Stable propagation of synchronous spiking in cortical neural networks. Nature 402:529-533. CrossRef Medline

Düring A, Coolen A, Sherrington D (1998) Phase diagram and storage capacity of sequence processing neural networks. J Phys A Math Gen 31: 8607-8621. CrossRef

Elizondo D (2006) The linear separability problem: some testing methods. IEEE Trans Neural Netw 17:330-344. CrossRef Medline

Gerstner W, Kistler WM (2002) Spiking neuron models: single neurons, populations, plasticity. New York: Cambridge UP.

Gerstner W, Ritz R, van Hemmen JL (1993) Why spikes? Hebbian learning and retrieval of time-resolved excitation patterns. Biol Cybern 69:503515. CrossRef Medline

Grossberg S (1969) On the serial learning of lists. Math Biosci 4:201-253. CrossRef

Hahnloser RH, Kozhevnikov AA, Fee MS (2002) An ultra-sparse code underlies the generation of neural sequences in a songbird. Nature 419:65-70. CrossRef Medline

Henneberger C, Papouin T, Oliet SH, Rusakov DA (2010) Long-term potentiation depends on release of $\mathrm{D}$-serine from astrocytes. Nature 463: 232-236. CrossRef Medline

Hertz J, Krogh A, Palmer RG (1991) Introduction to the theory of neural computation. Redwood City, CA: Addison-Wesley Longman.

Herz AVM, Sulzer B, Kühn B, Van Hemmen JL (1988) The Hebb rule: representation of static and dynamic objects in neural networks. Europhys Lett 7:663-669. CrossRef

Hinton G, Dayan P, To A, Neal R (1995) The Helmholtz machine through time. ICANN-95 483-490.

Hopfield JJ (1982) Neural networks and physical systems with emergent collective computational abilities. Proc Natl Acad Sci U S A 79:25542558. CrossRef Medline

Horn D, Levy N, Meilijson I, Ruppin E (2000) Distributed synchrony of spiking neurons in a Hebbian cell assembly. Adv Neural Inf Process Syst 12:129-135.

Lengyel M, Kwag J, Paulsen O, Dayan P (2005) Matching storage and recall: hippocampal spike timing-dependent plasticity and phase response curves. Nat Neurosci 8:1677-1683. CrossRef Medline

Lukoševičius M, Jaeger H (2009) Reservoir computing approaches to recurrent neural network training. Comput Sci Rev 3:127-149. CrossRef

Maass W, Markram H (2002) Synapses as dynamic memory buffers. Neural Netw 15:155-161. CrossRef Medline

Maass W, Sontag ED (2000) Neural systems as nonlinear filters. Neural Comput 12:1743-1772. CrossRef Medline

McCulloch WS, Pitts WH (1943) A logical calculus of the ideas immanent in nervous activity. Bull Math Biophys 5:115-133. CrossRef

Min R, Nevian T (2012) Astrocyte signaling controls spike timingdependent depression at neocortical synapses. Nat Neurosci 15:746753. CrossRef Medline

Mishchenko Y, Paninski L (2011) Efficient methods for sampling spike trains in networks of coupled neurons. Ann Appl Stat 5:1893-1919. CrossRef

Panatier A, Theodosis DT, Mothet JP, Touquet B, Pollegioni L, Poulain DA, 
Oliet SH (2006) Glia-derived D-serine controls NMDA receptor activity and synaptic memory. Cell 125:775-784. CrossRef Medline

Pfister JP, Gerstner W (2006) Triplets of spikes in a model of spike timingdependent plasticity. J Neurosci 26:9673-9682. CrossRef Medline

Pfister JP, Toyoizumi T, Barber D, Gerstner W (2006) Optimal spiketiming-dependent plasticity for precise action potential firing in supervised learning. Neural Comput 18:1318-1348. CrossRef Medline

Pillow J, Latham P (2008) Neural characterization in partially observed populations of spiking neurons. Adv Neural Inf Process Syst 20: 1161-1168.

Ponulak F, Kasiński A (2010) Supervised learning in spiking neural networks with resume: sequence learning, classification, and spike shifting. Neural Comput 22:467-510. CrossRef Medline

Rezende DJ, Wierstra D, Gerstner W (2011) Variational learning for recurrent spiking networks. Neural Inf Process Syst 24:136-144.

Rigotti M, Ben Dayan Rubin D, Wang XJ, Fusi S (2010) Internal representation of task rules by recurrent dynamics: the importance of the diversity of neural responses. Front Comput Neurosci 4:24. Medline
Shu Y, Hasenstaub A, Duque A, Yu Y, McCormick DA (2006) Modulation of intracortical synaptic potentials by presynaptic somatic membrane potential. Nature 441:761-765. CrossRef Medline

Sjöström PJ, Turrigiano GG, Nelson SB (2001) Rate, timing, and cooperativity jointly determine cortical synaptic plasticity. Neuron 32 : 1149-1164. CrossRef Medline

Sommer FT, Dayan P (1998) Bayesian retrieval in associative memories with storage errors. IEEE Trans Neural Netw 9:705-713. CrossRef Medline

Sompolinsky H, Kanter I I (1986) Temporal association in asymmetric neural networks. Phys Rev Lett 57:2861-2864. CrossRef Medline

Sutskever I, Hinton GE, Taylor G (2009) The recurrent temporal restricted Boltzmann machine. Adv Neural Inf Process Syst 21:1601-1608.

Xu S, Jiang W, Dan Y, Poo MM (2012) Activity recall in a visual cortical ensemble. Nat Neurosci 15:449-455, S1-S2. CrossRef Medline 